\title{
Application of SELDI-TOF mass spectrometry for the identification of differentially expressed proteins in transformed follicular lymphoma
}

\author{
Zhaosheng Lin ${ }^{1}$, Stephen D Jenson², Megan S Lim ${ }^{1,2}$ and Kojo SJ Elenitoba-Johnson ${ }^{1,2}$ \\ ${ }^{1}$ ARUP Institute for Clinical and Experimental Pathology and ${ }^{2}$ Department of Pathology, University of Utah \\ Health Sciences Center, Salt Lake City, UT, USA
}

\begin{abstract}
Completion of the human genome project has focused scientific attention on the development of methods that permit rapid characterization of proteins that are encoded by the genome. Recent improvements in twodimensional separation techniques in combination with protein identification software/databases and mass spectrometry (MS) now permit rapid comprehensive large-scale analysis of individual proteins within complex protein mixtures. We have performed pairwise comparisons of low-grade and transformed follicular lymphomas (FLs) in order to identify proteins that may be involved in FL progression using surface-enhanced laser desorption/ionization time-of-flight (SELDI-TOF) mass spectrometer (ProteinChip ${ }^{\mathrm{TM}}$, Ciphergen Biosystems). This system utilizes preactivated differential binding surfaces to achieve multidimensional chromatography. The protein-bound chips were then analyzed by a SELDI-TOF mass spectrometer to generate protein profiles. In preliminary experiments, we established that the MS data obtained from SELDI-TOF MS were reproducible, and that reduction in sample complexity improved the ability to detect lower abundance proteins. With specific regard to FL transformation, we rapidly identified a number of potential candidate proteins involved in this process. These included an upregulated $32 \mathrm{kDa}$ protein and a down-regulated $11.8 \mathrm{kDa}$ protein. Protein database searches revealed several candidates, among them cyclin D3 $(32.5 \mathrm{kDa})$ and caspase $3(11.8 \mathrm{kDa})$ whose differential expression were confirmed by immunoblotting and/or immunohistochemical analysis on the primary tissue specimens. Our studies demonstrate the utility of SELDI-TOF-MS for the rapid discovery of differentially expressed proteins using femtomolar quantities of crude protein derived from biopsy material. The versatility of this methodology supports its application to the rapid discovery of potential biomarkers in a variety of cellular systems.
\end{abstract}

Modern Pathology (2004) 17, 670-678 advance online publication, 16 April 2004; doi:10.1038/modpathol.3800100

Keywords: proteomics; mass spectrometry; SELDI; follicular lymphoma; transformation

Recent advances in the analytical separation of heterogeneous protein complexes and the development of robust and sensitive mass spectrometers capable of reliable analysis of peptides and proteins have strongly facilitated the global analysis of the protein complement of cells and tissues. Thus far, conventional methods of preanalytical sample purification such as liquid chromatography, immunoprecipitation, two-dimensional gel electrophoresis in combination with mass spectrometry (MS), have achieved only limited application in the discovery

Correspondence: KSJ Elenitoba-Johnson, Division of Anatomic Pathology, University of Utah Health Sciences Center, 50 North Medical Drive, Salt Lake City, UT 84132, USA.

E-mail: kojo.elenitobaj@path.utah.edu

Received 20 June 2003; revised 27 January 2004; accepted 04 February 2004; published online 16 April 2004 of biologic markers of disease. ${ }^{1}$ This limited role can be explained in part by the demanding nature of preanalytical techniques such as high-performance liquid chromatography and capillary electrophoresis, as well as difficulties in the reproducible performance of two-dimensional gel electrophoresis to high levels of technical proficiency.

New strategies that facilitate proteomic analysis by dramatically simplifying the preanalytical sample separation and coupling with MS have recently been introduced for biomarker discovery research. ${ }^{2}$ One such strategy is the surface-enhanced laser desorption/ionization (SELDI) which has recently been combined with time-of-flight (TOF) MS in a commercialized embodiment known as the ProteinChip ${ }^{\mathrm{TM}}$ Array System (Ciphergen ${ }^{\circledR}$ Biosystems, Fremont, CA, USA). The system consists of ProteinChip Arrays, a mass analyzer and sophisticated 
software for data collection and analysis. ${ }^{3,4}$ Each chip contains a strip of 8-16 spots, which take advantage of the unique physicochemical properties of proteins for the achievement of differential separation and chromatography. Different spots in the biochip carry distinctive chromatographic properties such as hydrophobic, hydrophilic, ion exchange and immobilized metallic ion matrices, which have been traditionally exploited for differential separation of proteins. In addition, affinity capture using specific functional groups such as DNA, receptors, and antibodies covalently bound to the matrices permits specific isolation of proteins with unique binding properties to the selected surfaces. $^{3,4}$ The requirement of minimal amounts of tissue and the ability to analyze crude protein extracts make this platform attractive for studying tissue biopsy material.

In this study, we have applied SELDI-TOF/MS for the identification of differentially expressed proteins potentially involved in follicular lymphoma (FL) transformation. Total protein was extracted from archival frozen tissue samples consisting of two matched pairs of FL and their corresponding transformed FLs (diffuse large B-cell lymphoma) (DLBCL) counterparts occurring in the same individuals. The crude protein lysates were applied directly to the ProteinChip ${ }^{\mathrm{TM}}$ system and without preanalytical separation, subjected to SELDI-TOF/ MS. The mass spectral profiles of the samples were compared, and by combining the molecular weight, isoelectric point (PI) and chip affinity characteristics at which maximal differential expression was observed, with focused database searching, we successfully identified cyclin D3 and caspase 3 as the differentially expressed proteins in the DLBCLs. Our studies show the powerful potential of MS for the rapid discovery of tumor biomarkers and proteins that may be relevant to the pathogenesis of disease processes.

\section{Materials and methods}

\section{Tissue Samples}

Frozen tissue biopsy specimens consisting of matched pairs of FL and the DLBCL counterparts from two patients were obtained from the archives of the Hematopathology Section of the University of Utah Health Sciences Center, Salt Lake City, Utah. The diagnoses were made according to the World Health Organization classification ${ }^{5}$ of lymphoid neoplasms and were confirmed by independent review by two hematopathologists (MSL and KSJE-J).

\section{Preparation of Cell Lysates and Western Blot Analysis}

Tissues were ground in liquid nitrogen and then lysed in lysis buffer (1\% Triton X-100, $10 \mathrm{mM} \mathrm{NaCl,}$
$10 \mathrm{mM}$ Tris-HCl pH 8.6). containing $0.1 \%$ protease inhibitor cocktail (Sigma Chemicals, St Louis, MO, USA). Cell membranes and other undissolved components were removed by micro-centrifugation at top speed for $10 \mathrm{~min}$ at $4^{\circ} \mathrm{C}$. A measure of $15 \mu \mathrm{g}$ of total protein extracts from each sample were resolved in a $12 \%$ sodium-dodecyl sulfate-polyacrylamide gel (SDS-PAGE) using a BioRad mini trans-blot system (Biorad, Hercules, CA, USA). Following SDS-PAGE, the proteins were transferred onto a nitrocellulose membrane using the semi-dry transfer apparatus (BioRad, Hercules, CA, USA). Western blot analysis was performed using the following antibodies; mouse anti-human monoclonal antibody against cyclin D3 (Research Diagnostics, Inc., Flanders, NJ, USA), and polyclonal antibody against caspase 3 (Cell signaling, Beverly, MA, USA). Immunoreactive protein bands were visualized using the enzyme chemiluminescence detection kit (Amersham, Arlington Heights, IL, USA).

\section{SELDI-TOF Analysis}

We analyzed crude protein lysates from reactive lymphoid tissues including reactive tonsils $(n=2)$ low-grade FLs $(n=2)$ and their transformed counterparts-DLBCL $(n=2)$ arising in the same individual. The instrument readout for each sample was visualized as a mass spectrum composed of peaks from 3 to $100 \mathrm{kDa}$. The mass spectral pattern for each sample consisted of several peaks whose mass/ charge ratios were depicted on the $x$-axis, while the height of the peak (relative abundance) was disposed along the $y$-axis. An additional cohort of FLs $(n=5)$ and DLBCL $(n=5)$ were selected for validation of MS results by Western blotting analysis. The abundance levels of the different proteins represented by the $\mathrm{m} / \mathrm{z}$ peaks were alternatively displayed as 'virtual Western blots', which are digital representations of the area under the curves of each peak within a mass spectrum.

\section{Sample Loading and Washing}

A measure of $2 \mu \mathrm{g}$ of protein per sample were loaded onto each spot in duplicate. Before samples were analyzed by the SELDI-TOF MS, loaded chips were washed twice using one of the following buffers depending on what chip surface the sample was loaded on: PBS or PBS containing $1 \mathrm{M} \mathrm{NaCl}$ for SAX (strong anion exchanger) chips; deionized water or $50 \%$ acetonitrile for hydrophobic chips; and PBS containing $0.5 \mathrm{M} \mathrm{NaCl}$ or PBS containing $25 \mathrm{mM}$ imidazole for IMAC (immobilized metal affinity capture) chips. After sample loading, the protein bound chips were transferred into the TOF mass spectrometer (Ciphergen, Fremont, CA, USA) and the molecular weights were scanned from 5 to $50 \mathrm{kDa}$. The laser intensity was adjusted depending 
on the signal to noise ratio of the mass peak heights to background. The spectra were generated at using signal averaging 90 laser shots. Analysis of the mass spectra was performed using the ProteinChip software, PEAKS 2.0.1. (Ciphergen, Fremont, CA, USA). We utilized bovine insulin $(5.7336 \mathrm{kDa})$, bovine serum albumin $(66.410 \mathrm{kDa})$, and bovine cytochrome $C(12.230 \mathrm{kDa})$ molecular weight standards as external calibrators. Protein candidates were identified by using the SWISS-PRO search program and database: www.expasy.ch/tools/tagident.html.

\section{Immunohistochemical Studies}

Immunohistochemistry was performed on $5 \mu \mathrm{m}$ thick paraffin-embedded tissue sections mounted on silanated glass slides. The tissue sections were prepared for antibody application by heat-induced epitope retrieval as follows: citrate buffer ( $\mathrm{pH}$ 6.0) or (TRIS $\mathrm{pH}$ 9.5) for $3 \mathrm{~min}$. Immunostaining was performed using an avidin-biotin peroxidase complex technique on automated immunostainer (Ventana Medical System, Tucson, AZ, USA). Color development was achieved using diaminobenzidine as the chromogen. The following panel of monoclonal antibodies was utilized: L26 (CD20) (Dako, Carpenteria, CA, USA), CD3 (Novocastra, Newcastle upon Tyne, UK), BCL-2 (Dako, Carpenteria, CA, USA), BCL-6 (Santa Cruz Biotechnology, Santa Cruz, CA, USA), CD10 (Novocastra, Newcastle upon Tyne, UK), and cyclin D3 (Research Diagnostics, Flanders, NJ, USA) and caspase 3 (Cell signaling, Beverly, MA, USA). Slides were counterstained with hematoxylin, dehydrated and coverslipped with a permanent mounting medium.

\section{Immunoglobulin Heavy Chain Polymerase Chain Reaction (IgH-PCR)}

We performed IgH-PCR analysis to evaluate clonal relationships between the initial FL and the transformed (DLBCL) samples on both patient samples. Clonal bands of identical size between samples obtained from one matched pair are suggestive of a clonal relationship. IgH-PCR analysis was performed using a 1605 Rapid Air Thermocycler (Rapid cycler, Idaho Technology, Idaho Falls, ID). A heminested approach was employed using the VHA-FRIII (5' ACA CGG C(C/T)G T(G/A)T ATT ACT GT 3', bp 555-574 GenBank Accession \#M29812) immunoglobulin heavy chain V-region primer and the Jha primer ( $5^{\prime}$ ACC TGA GGA GAC GGT GAC C $3^{\prime}$, bp 2523 to 2541 GenBank Accession \#X86359) for the first round of PCR. The first round of PCR involved 25 cycles of denaturation at $94^{\circ} \mathrm{C}$ for $0 \mathrm{~s}$, annealing at $55^{\circ} \mathrm{C}$ for $0 \mathrm{~s}$, and extension at $72^{\circ} \mathrm{C}$ for $20 \mathrm{~s}$. A volume of $1 \mu \mathrm{l}$ of the resulting amplification product was utilized as the template in the subsequent heminesting reaction which incorporated an internal $\mathrm{JH}$ primer (5’ GTG ACC AGG GT(G/T/A/C) CCT TGG
CCC CAG 3', bp 302-325 GenBank Accession \#X86359) in 30 cycles of denaturation at $94^{\circ} \mathrm{C}$ for $0 \mathrm{~s}$, annealing at $50^{\circ} \mathrm{C}$ for $0 \mathrm{~s}$ and extension at $72^{\circ} \mathrm{C}$ for $10 \mathrm{~s}$. Amplifications were carried out in a $10 \mu \mathrm{l}$ glass capillary containing $50 \mathrm{mmol}$ TRIS ( $\mathrm{pH} 8.5$ ), $3.0 \mathrm{mmol} / \mathrm{l} \mathrm{MgCl}_{2}$, four deoxynucleotide triphosphates at $200 \mu \mathrm{mol} / \mathrm{l}$ each, primers at $0.5 \mu \mathrm{mol} / \mathrm{l}$ and $0.4 \mathrm{U}$ Taq DNA polymerase (Promega, Madison, WI, USA). PCR products were run on $1.5 \%$ agarose gels (FMC Bioproducts, Rockland, ME, USA) and detected with ethidium-bromide staining and by ultra-violet transillumination.

\section{Results}

\section{IgH-PCR}

The FLs and their corresponding DLBCL counterparts yielded clonal bands of identical size when evaluated by IgH-PCR (data not shown).

\section{SELDI-TOF MS}

Mass spectrometric data obtained from crude biopsy samples are reproducible

We subjected crude cell lysates from archival frozen tissue samples to direct analysis by MS using SELDI-TOF. The patterns obtained were reproducible for a given sample, and common features were identifiable among similar samples. Mass spectral profiles and virtual Western blots illustrate the consistent reproducibility of SELDI-TOF MS analysis using crude protein lysates (Figure 1). Figure 1 shows a consistent mass spectrum in replicate

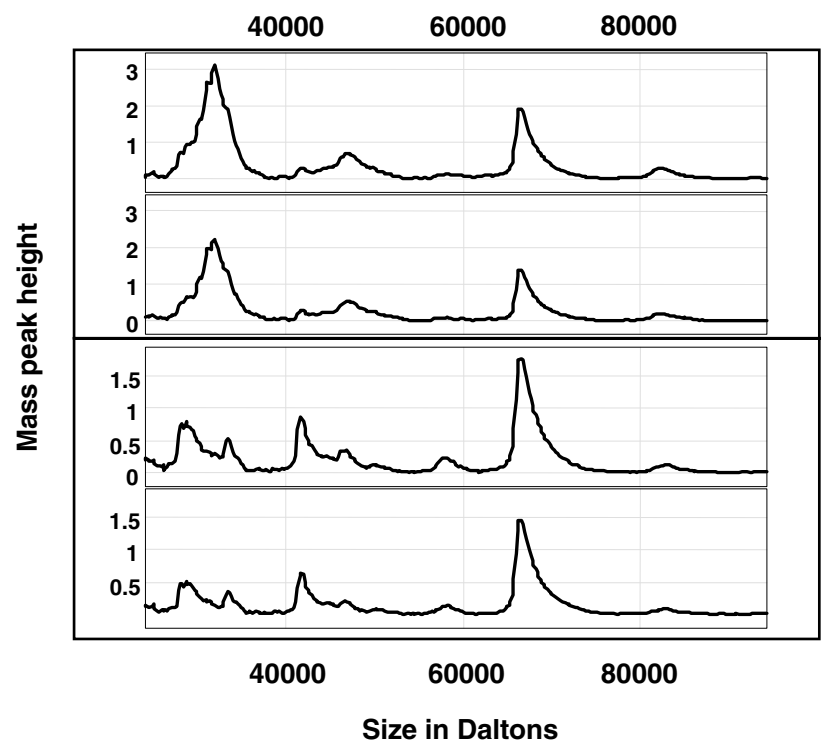

Figure 1 Reproducibility of SELDI-TOF MS in the proteomic analysis of crude lymphoma cell lysates. The patterns obtained were reproducible for a given sample. The mass spectral profiles show duplicate analyses of a FL sample (upper panels), and a diffuse large B-cell lymphoma sample (lower panels). 
analyses of crude protein extracts from FL\#1 (upper panel). Similarly, replicate analyses of a DLBCL (sample \#2) lower panel, showed reproducible mass profiles in replicate experiments. The mass spectra for the DLBCL were distinct from that of the FL.

\section{Reduction in sample complexity improves}

sensitivity of detection of minor components

Utilization of purification columns that discriminate between proteins of specific sizes improved the detection of protein components that were present in lower quantities than the major constituents. In Figure 2a, we show that by using size-fractionation columns that select for proteins $\leq 30 \mathrm{kDa}$, we improved our ability to detect lower molecular weight proteins that were barely discernable using the whole cell lysates. We utilized three different chip surfaces with distinct chromatographic properties and differential binding affinities for proteins with specific biophysical characteristics. The H4 chip exhibits a matrix with affinity for hydrophobic proteins. The immobilized metallic affinity column (IMAC) surface exhibits affinity for phosphorylated proteins, and proteins with acidic moieties. The strong anionic exchange (SAX) surface selects for proteins at $\mathrm{pH}$ higher than their pIs. Figure $2 \mathrm{~b}$ and $\mathrm{c}$ shows that different chromatographic parameters, affinity characteristics and modulation of washing stringencies can influence the detection of specific proteins.

Protein profiling of matched pairs of low-grade FL and their transformed counterparts (DLBCL) reveals differentially expressed proteins

We compared the mass spectral profiles of our matched pairs of low-grade FL and DLBCL and identified consistent differences corresponding to differentially expressed proteins. The SAX surface yielded the most readily discernable mass/charge $(\mathrm{m} / \mathrm{z})$ peaks that were distinguishable above background. In addition, the best resolution $\mathrm{m} / \mathrm{z}$ peaks was observed between 5 and $30 \mathrm{kDa}$. Our studies revealed that a $32 \mathrm{kDa}$ protein was upregulated and an $11.8 \mathrm{kDa}$ protein was downregulated in both of our DLBCLs, when compared to their corresponding

Figure 2 Reduction in sample complexity improves detection of constituent components of complex protein mixtures. (a). Size fractionation columns improve the spectral resolution and the detection of proteins in selected size ranges. The K30 sizing column markedly improves the detection of proteins in the 20$50 \mathrm{kDa}$ size range. (b). Different chromatographic parameters select for different proteins. Numerous mass peaks are accentuated or obliterated depending on the chromatographic surface employed. IMAC represents immobilized metal affinity column. $\mathrm{H} 4$ represents hydrophobic surface. SAX represents the strong anionic exchange surface. (c). Specific protein capture and retention can be achieved with modulation of washing stringency. For example, phosphate-buffered saline revealed a greater variety of proteins than phosphate-buffered saline (PBS) and $25 \mathrm{mM}$ imidazole (Imdz).
FLs (Figure 3). We incorporated both molecular weight information with the $\mathrm{pI}$ and chip binding characteristics for the identification of cyclin D3 $(32.5 \mathrm{kDa})$ and caspase $3(11.8 \mathrm{kDa})$ as the differentially expressed proteins.
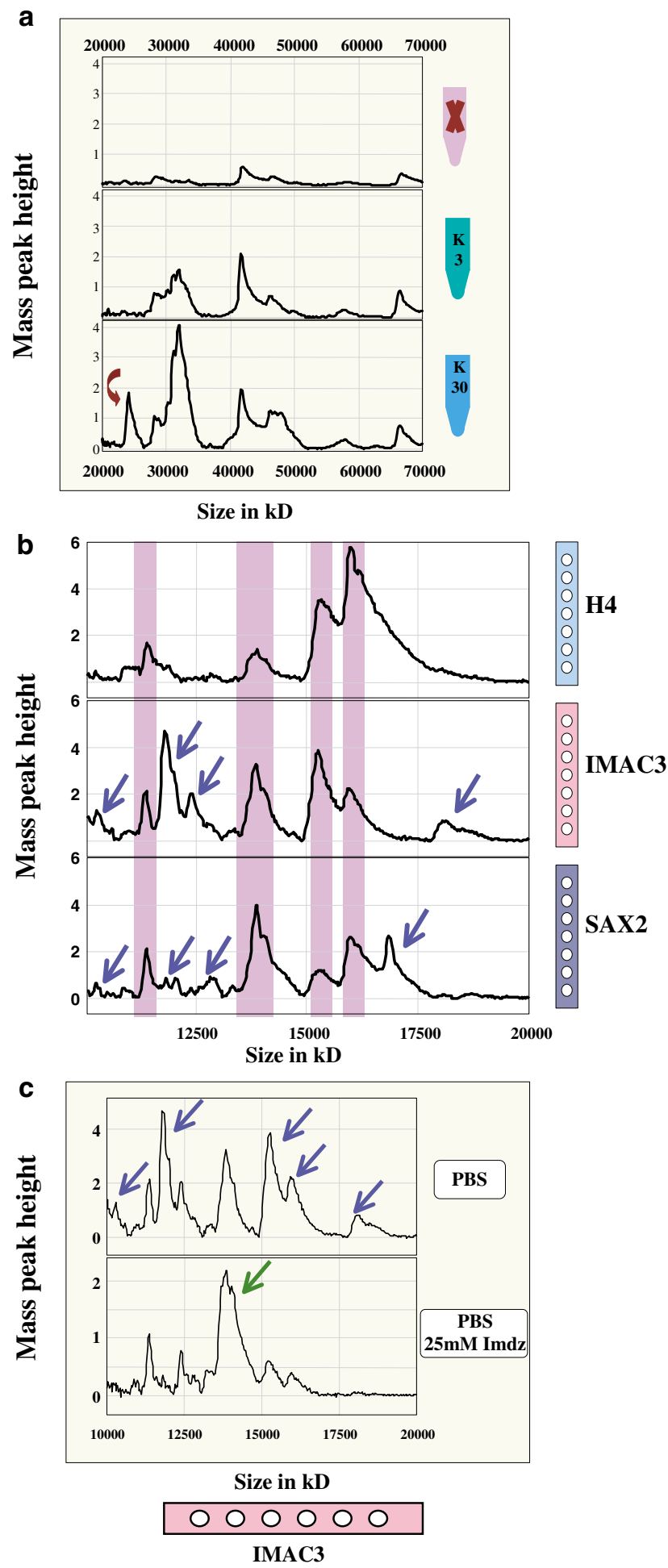

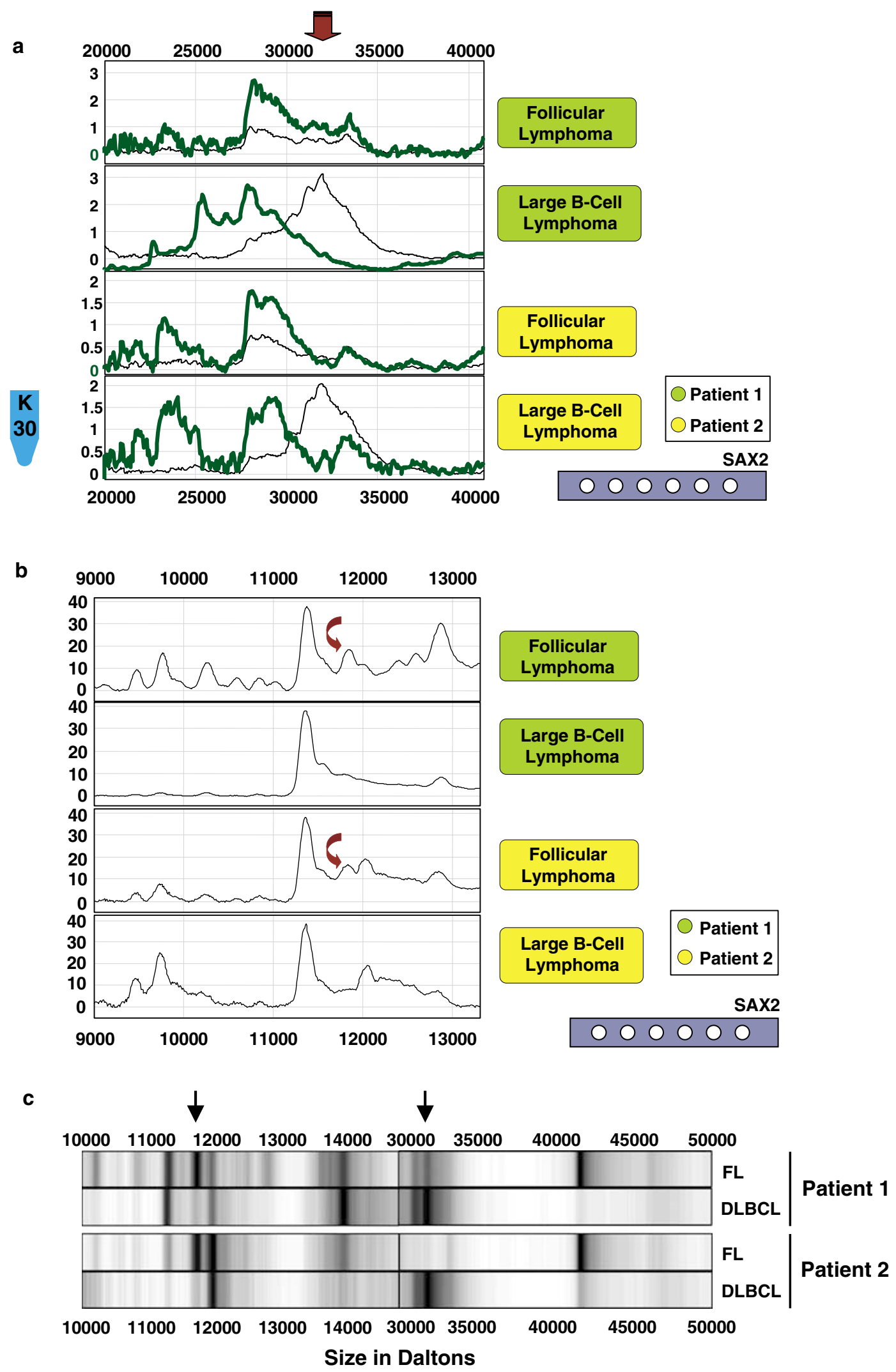

Figure 3 SELDI-TOF MS spectra of matched pairs of transformed FL. Two matched pairs of FL and their transformed DLBCL counterparts were assessed in parallel and mass spectral profiles superimposed. Analysis of the mass spectrographic profiles generated using the strong cationic exchange/immobilized metallic affinity-capture surface chemistry reveals upregulation of a $32 \mathrm{kDa}$ protein (a) and downregulation of an $11.8 \mathrm{kDa}$ protein (b) in the transformed DLBCL samples. (c) Shows virtual Western blots of (a) and (b). 


\section{Western Blotting and Immunohistochemistry}

Differential expression identified by MS is corroborated by Western blotting and tissue immunohistochemistry

All of the lymphoma samples studied demonstrated positive immunohistochemical reactivity for CD20, BCL-2, BCL-6 and CD10. The neoplastic cells in all cases were uniformly negative for CD3. In order to confirm the differential expression identified by MS, we performed immunoblotting analysis using antibodies specific for cyclin D3. Figure 4a shows immunoblotting analysis with higher levels of cyclin D3 in the transformed DLBCLs when compared to the FLs. We also studied five FLs and five transformed DLBCLs (unrelated) by Western blotting and observed comparatively higher levels of cyclin D3 in the DLBCLs (Figure 4b). Upregulation of cyclin D3 was confirmed by tissue immunohistochemistry (Figure 4c), in which increased nuclear signals for cyclin D3 were detectable in the DLBCLs, whereas their low-grade FL counterparts showed only rare positive signals. In concordance with the results of SELDI-TOF MS, Western blot and immunohistochemical studies showed decreased expression of caspase 3 in the DLBCLs compared with their FL's counterparts from the same patients (Figures $4 \mathrm{~d}$ and 5).

\section{Discussion}

Proteomic studies have traditionally involved a combination of two-dimensional gel electrophoresis and MS coupled with mass spectrometry. Twodimensional gel electrophoresis is currently the most extensively utilized methodology for proteomic studies. In this approach, proteins are first separated based on their pI in the first dimension, and then by their molecular weights in the second dimension. While this method is powerful and possesses a high-resolution capacity, it is limited by its under-representation of proteins at both extremes of molecular weight and pI. In addition, two-dimensional gel electrophoresis is labor-intensive, requires large quantities of intact protein for optimal performance and technically difficult to perform with high reproducibility. All of these factors have limited its routine application in the evaluation of human tissue biopsy material. Hence, a number of innovative approaches which address the problems associated with two-dimensional gel electrophoresis-based approaches have recently been developed for proteomic studies., ${ }^{3,6,7}$ A promising development in high-throughput proteomics is the advent of protein bio-chip technology. ${ }^{3}$

Protein chip biotechnology represents an example of a promising approach that facilitates high throughput proteomics analyses. Protein bio-chips for proteomic analysis currently exist as either immobilized recognition molecules (antibodies or aptamers) or in formats wherein the biophysical or biochemical interaction properties of the protein are exploited for protein isolation in combination with MS. This latter format is embodied in the Protein Chip ${ }^{\circledR}$ system (Ciphergen, Fremont, CA, USA), which includes several different chromatographic surfaces that capture different subclasses of proteins. For SELDI-TOF analysis, proteins isolated from samples of interest are applied directly to the chip surfaces. After preferential binding of specific proteins on the chip surface, the proteins are detected by a TOF mass spectrometer fitted with a laser desorption ion-source. ${ }^{3}$ The sample loaded onto the chip surface is subjected to laser activation from a pulse emanating from a laser source. The energy absorbing matrix molecules within the chip cocrystalize with the adsorbed proteins. Laser energy absorbed by the sample leads to increased thermal energy with consequent desorption of ions from the chip surface. The matrix transfers laser energy into kinetic energy, which facilitates the travel of the ionized proteins to the detector. Laser desorption ionization occurs when laser energy absorbed by the sample leads to increased thermal energy with consequent desorption of ions from the biochip surface. The charged particles (ions) travel through an electromagnetic field within which they are accelerated until they impact the detector. The smaller ions are accelerated more rapidly and have a shorter flight time as compared to the heavier ions. This relationship between the mass of the ion and its time of flight is used to determine the mass of the charged particles.

SELDI-TOF MS offers several advantages in the analysis of tissue biopsies. Preanalytical sample preparation is rapid and dramatically simplified because of the ability to achieve chromatographic separation using the specialized chip surfaces that bind proteins based on their differential physicochemical characteristics. ${ }^{8,9}$ Of particular relevance to the proteomic assessment of tissue biopsy material is the exquisite sensitivity of SELDI-TOF MS and thus its attendant requirement for miniscule quantities of sample. The platform is amenable to the evaluation of crude protein lysates derived from crude biologic material such as tissue biopsies and biologic fluids. ${ }^{4,10-12}$ On the other hand, SELDI-TOF MS suffers from the drawback of inability to provide immediate and direct identification of the differentially expressed proteins, so additional postanalytical strategies have to be employed for the identification of the potential biomarkers.

We have taken advantage of the intrinsic advantages of SELDI-TOF MS in our studies aimed at identifying deregulated proteins involved in FL transformation. Our studies revealed upregulation of cyclin D3 in the transformed FLs (DLBCLs). This finding is interesting since, deregulated expression of the proteins involved in the cell cycle is a common mechanism implicated in tumorigenesis. ${ }^{13}$ The D-cyclins (cyclin D1, D2, D3) form complexes with cyclin-dependent kinases 4 and 6, which 
676

a

\title{
Patient 1 Patient 2
}
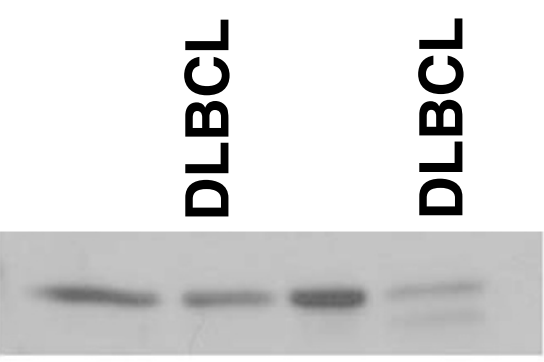

\section{Caspase 3}

\author{
$\beta$-actin
}

b

Western Blot

\section{0 unmatched cases DLBCL}

Cyclin D3

B-actin

C

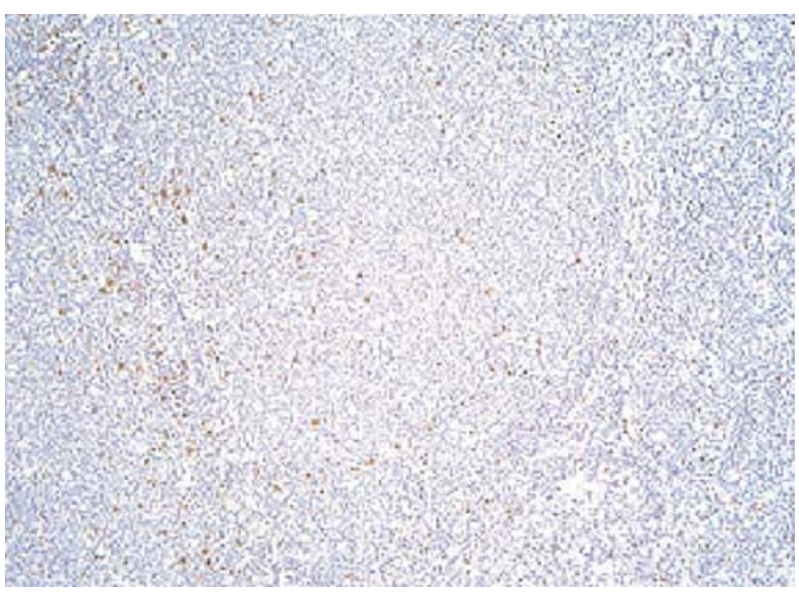

FL

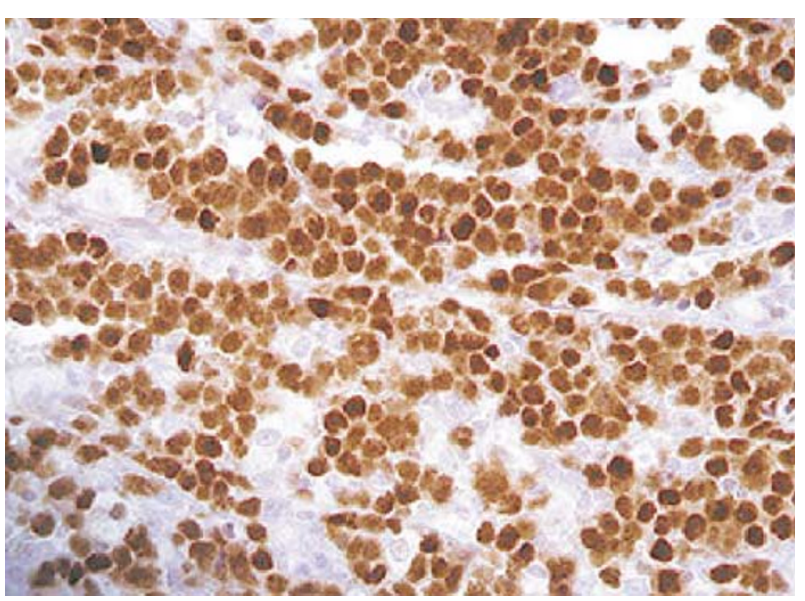

DLBCL

Figure 4 Demonstration of cyclin D3 overexpression and caspase 3 downregulation in transformed follicular lymphoma. (a) and (b). Western blotting analysis using antibodies specific to human cyclin D3 shows higher levels of expression in the DLBCLs in comparison to the related FLs (a) and unrelated FLs (b). Antibody reactivity against $\beta$-actin served as an appropriate loading control. (c). Photomicrograph of immunohistochemistry for cyclin D3 shows minimal expression in follicular lymphoma (case 1), and the corresponding DLBCL counterpart from patient 1 shows high levels of nuclear cyclin D3 in the tumor cells. (d). Western blot analysis for caspase 3 expression in transformed FL. Western blotting using antibodies specific to caspase 3 shows higher levels of caspase 3 expression in the transformed DLBCL counterparts. Antibody reactivity against $\beta$-actin served as an appropriate loading control. 

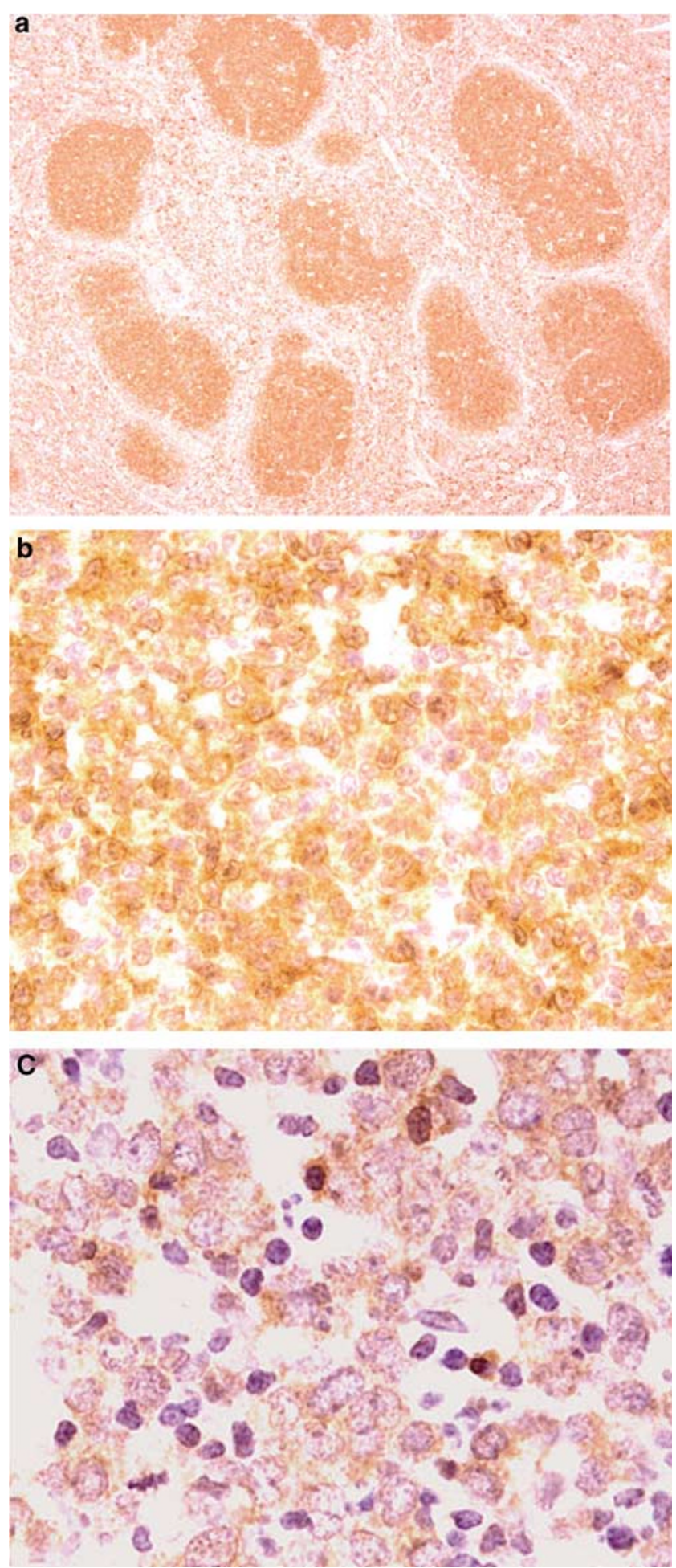

Figure 5 Immunohistochemical demonstration of caspase 3 downregulation in transformed FL. Top panel. Caspase 3 expression is observed in neoplastic follicles of a case of FL. Middle panel. Higher magnification photomicrograph showing high levels of caspase 3 in the cytoplasm of the FL cells. Bottom panel. Decreased caspase 3 signals in the cytoplasm of the DLBCL arising from the FL illustrated in the top and middle panels.

phosphorylate and inactivate the retinoblastoma protein, thereby promoting cell cycle progression from G1 to the S-phase. ${ }^{14}$ The prototype for deregulated expression of a cyclin $\mathrm{D}$ protein is typified by cyclin D1, which is overexpressed in mantle cell lymphoma as a consequence of the $\mathrm{t}(11 ; 14) .{ }^{15,16}$ The related protein cyclin D3 has been shown to exert a growth-promoting effect in lymphoid cells, as well as induction and maintenance of terminal differentiation in nonlymphoid tissues. ${ }^{14}$ High cyclin D3 expression has been identified as a predictor of poor response to chemotherapy, poor outcome and short overall survival independent of the International Prognostic Index in patients with DLBCL. ${ }^{17}$ As in other studies, we did not observe a significant correlation between cyclin D3 expression and proliferative index as measured by reactivity with the MIB-1 antibody (data not shown). This indicates that the high cyclin D3 overexpression is not a direct consequence of the increased proliferative state observed in DLBCL when compared to FL. To our knowledge, previous studies have not shown involvement of cyclin D3 upregulation in FL transformation.

Our studies also revealed downregulation of caspase 3 in the transformed FLs (DLBCLs). This is interesting since subversion of apoptosis is a common mechanism exploited in tumorigenesis. ${ }^{18-20}$ The caspase (cysteine aspartic acid proteases) family of proteins are important mediators of apoptosis. $^{21}$ Of these, caspase 3 expression in particular appears to be best correlated with apoptosis. ${ }^{22}$ Further, it has recently been shown that BCL-2 and its close homolog BCL- $\mathrm{X}_{\mathrm{L}}$ are inhibitors of activation of caspase $3 .^{23-25} \mathrm{~A}$ physiologic role for caspase 3 has been described wherein CD40mediated germinal center stimulation has also been shown to involve suppression of caspase 3 expression and upregulation of BCL-2 and BCL-2 $\mathrm{X}_{\mathrm{L}}$, concertedly providing protection from anti-Ig-induced apoptosis. ${ }^{26,27}$ Reactive germinal center B cells exhibit high levels of caspase 3 expression, whereas the long-lived mantle $B$-cells exhibit dramatically lower or absent levels of caspase $3 .^{22}$ Thus it is plausible that deregulation of caspase 3 is a plausible contributory mechanism for neoplastic transformation. Malignant lymphomas demonstrate a variety of patterns of caspase 3 expression depending on the specific subtype. Specifically, low-grade (Grade I) FLs reportedly exhibit low levels of caspase 3 expression, while grade III FLs show a high levels of caspase 3 protein expression in majority of cases. ${ }^{22}$ By comparison, DLBCLs demonstrate a widely variable pattern with high expression in some cases, and low to absent levels in others. ${ }^{2,28}$ Our studies demonstrate a role for down regulation of caspase 3 in a subset of transformed FL lymphomas.

In summary, we have utilized SELDI-TOF MS in the identification of deregulated proteins involved in FL transformation. The ability to rapidly profile the expression of several proteins across the proteome greatly facilitates identification multiple deregulated proteins with great potential for eluci- 
dation of pathways that are deregulated in the pathogenesis of specific disease entities. In this study, our data implicate the simultaneous deregulation of the cell cycle and apoptosis-related proteins in FL transformation. Potential improvements for this technology include increased variety of affinity capture surfaces that permit better resolution of proteins and incorporation of algorithms that permit direct identification of proteins. Nevertheless, our results demonstrate the power and potential of SELDI-TOF mass spectrometry for the rapid discovery of novel biomarkers relevant to the pathogenesis of tumor progression.

\section{Acknowledgements}

We are grateful to Gongyi Shi and Mark Smith of Ciphergen, Fremont, CA.

\section{References}

1 Liotta LA, Kohn EC, Petricoin EF. Clinical proteomics: personalized molecular medicine. JAMA 2001;286: 2211-2214.

2 Fung ET, Wright Jr GL, Dalmasso EA. Proteomic strategies for biomarker identification: progress and challenges. Curr Opin Mol Ther 2000;2:643-650.

3 Merchant M, Weinberger SR. Recent advancements in surface-enhanced laser desorption/ionization-time of flight-mass spectrometry. Electrophoresis 2000;21: 1164-1177.

4 Chapman K. The ProteinChip Biomarker System from Ciphergen Biosystems: a novel proteomics platform for rapid biomarker discovery and validation. Biochem Soc Trans 2002;30:82-87.

5 Harris NL, Jaffe ES, Diebold J, et al. World Health Organization classification of neoplastic diseases of the hematopoietic and lymphoid tissues: report of the Clinical Advisory Committee meeting-Airlie House, VA, November 1997. J Clin Oncol 1999;17:3835-3849.

6 Link AJ, Eng J, Schieltz DM. Direct analysis of protein complexes using mass spectrometry. Nat Biotechnol 1999;17:676-682.

7 Gygi SP, Rist B, Gerber SA, et al. Quantitative analysis of complex protein mixtures using isotope-coded affinity tags. Nat Biotechnol 1999;17:994-999.

8 Paweletz CP, Trock B, Pennanen M, et al. Proteomic patterns of nipple aspirate fluids obtained by SELDITOF: potential for new biomarkers to aid in the diagnosis of breast cancer. Dis Markers 2001;17: 301-307.

9 Issaq HJ, Veenstra TD, Conrads TP, et al. The SELDITOF MS approach to proteomics: protein profiling and biomarker identification. Biochem Biophys Res Commun 2002;292:587-592.

10 Fetsch PA, Simone NL, Bryant-Greenwood PK, et al. Proteomic evaluation of archival cytologic material using SELDI affinity mass spectrometry: potential for diagnostic applications. Am J Clin Pathol 2002;118: 870-876.
11 Vlahou A, Schellhammer PF, Mendrinos S, et al. Development of a novel proteomic approach for the detection of transitional cell carcinoma of the bladder in urine. Am J Pathol 2001;158:1491-1502.

12 Petricoin EF, Ardekani AM, Hitt BA, et al. Use of proteomic patterns in serum to identify ovarian cancer. Lancet 2000;359:572-577.

13 Sherr CJ. Cell cycle control and cancer. Harvey Lect 2000;96:73-92.

14 Bartkova J, Lukas J, Strauss M, et al. Cyclin D3: requirement for G1/S transition and high abundance in quiescent tissues suggest a dual role in proliferation and differentiation. Oncogene 1998;17:1027-1037.

15 Williams ME, Westermann CD, Swerdlow SH. Genotypic characterization of centrocytic lymphoma: frequent rearrangement of the chromosome 11 bcl-1 locus. Blood 1990;76:1387-1391.

16 Rimokh R, Berger F, Bastard C, et al. Rearrangement of CCND1 (BCL1/PRAD1) $3^{\prime}$ untranslated region in mantle-cell lymphomas and $\mathrm{t}(11 \mathrm{q} 13)$-associated leukemias. Blood 1994;83:3689-3696.

17 Filipits M, Jaeger U, Pohl G, et al. Cyclin D3 is a predictive and prognostic factor in diffuse large B-cell lymphoma. Clin Cancer Res 2002;8:729-733.

18 Reed JC. Mechanisms of apoptosis avoidance in cancer. Curr Opin Oncol 1999;11:68-75.

19 Lee JM, Bernstein A. Apoptosis, cancer and the p53 tumour suppressor gene. Cancer Metastasis Rev 1995; 14:149-161.

20 Kaufmann SH, Gores GJ. Apoptosis in cancer: cause and cure. Bioessays 2000;22:1007-1017.

21 Dixit VM. Role of ICE-proteases in apoptosis. Adv Exp Med Biol 1996;406:113-117.

22 Krajewski S, Gascoyne RD, Zapata JM, et al. Immunolocalization of the ICE/Ced-3-family protease, CPP32 (Caspase-3), in non-Hodgkin's lymphomas, chronic lymphocytic leukemias, and reactive lymph nodes. Blood 1997;89:3817-3825.

23 Armstrong RC, Aia T, Xiang J, et al. Fas-induced activation of the cell death-related protease CPP32 Is inhibited by Bcl-2 and by ICE family protease inhibitors. J Biol Chem 1996;271:16850-16855.

24 Boulakia CA, Chen G, Ng FW, et al. Bcl-2 and adenovirus EIB $19 \mathrm{kDa}$ protein prevent E1A-induced processing of CPP32 and cleavage of poly(ADP-ribose) polymerase. Oncogene 1996;12:529-535.

25 Chinnaiyan AM, Orth K, O'Rourke K, et al. Molecular ordering of the cell death pathway. Bcl-2 and Bcl-xL function upstream of the CED-3-like apoptotic proteases. J Biol Chem 1996;271:4573-4576.

26 An S, Knox KA. Ligation of CD40 rescues RamosBurkitt lymphoma B cells from calcium ionophoreand antigen receptor-triggered apoptosis by inhibiting activation of the cysteine protease CPP32/Yama and cleavage of its substrate PARP. FEBS Lett 1996;386: 115-122.

27 Liu YJ, Mason DY, Johnson GD, et al. Germinal center cells express bcl-2 protein after activation by signals which prevent their entry into apoptosis. Eur J Immunol 1991;21:1905-1910.

28 Donoghue S, Baden HS, Lauder I, et al. Immunohistochemical localization of caspase-3 correlates with clinical outcome in B-cell diffuse large-cell lymphoma. Cancer Res 1999;59:5386-5391. 